\title{
ANOPP Landing Gear Noise Prediction Comparisons to Model-Scale Data
}

\author{
Casey L. Burley ${ }^{*}$, Thomas F. Brooks ${ }^{\dagger}$ and William M. Humphreys, Jr. * \\ NASA Langley Research Center, Hampton, Virginia 23681 \\ John W. Rawls, Jr. ${ }^{\S}$ \\ Lockheed Martin Space Operations Langley Program Office, Hampton, Virginia 23681
}

\begin{abstract}
The NASA Aircraft NOise Prediction Program (ANOPP) includes two methods for computing the noise from landing gear: the "Fink" method and the "Guo" method. Both methods have been predominately validated and used to predict full-scale landing gear noise. The two methods are compared, and their ability to predict the noise for model-scale landing gear is investigated. Predictions are made using both the Fink and Guo methods and compared to measured acoustic data obtained for a high-fidelity, 6.3\%-scale, Boeing 777 main landing gear. A process is developed by which full-scale predictions can be scaled to compare with model-scale data. The measurements were obtained in the NASA Langley Quiet Flow Facility for a range of Mach numbers at a large number of observer polar (flyover) and azimuthal (sideline) observer angles. Spectra and contours of the measured sound pressure levels as a function of polar and azimuthal angle characterize the directivity of landing gear noise. Comparisons of predicted noise spectra and contours from each ANOPP method are made. Both methods predict comparable amplitudes and trends for the flyover locations, but deviate at the sideline locations. Neither method fully captures the measured noise directivity. The availability of these measured data provides the opportunity to further understand and advance noise prediction capabilities, particularly for noise directivity.
\end{abstract}

\section{Nomenclature}

$\begin{array}{ll}\text { c } & =\text { ambient speed of sound } \\ D & =\text { directivity function } \\ d & =\text { wheel diameter } \\ F & =\text { spectrum shape function } \\ f & =\text { frequency, Hz } \\ L & =\text { strut length } \\ M & =\text { freestream Mach number } \\ N_{w} & =\text { number of wheels per landing gear assembly } \\ P & =\text { narrowband component spectrum (Guo method) } \\ \left\langle p^{2}\right\rangle & =\text { acoustic mean-square pressure } \\ R & =\text { source-to-observer distance } \\ S_{t} & =\text { Strouhal number } \\ \mathrm{S} & =\text { aggregate surface integration parameter (Guo method) } \\ W & =\text { aircraft weight }\end{array}$

\footnotetext{
* Senior Research Scientist, Aeroacoustics Branch, MS 461, Member AIAA.

${ }^{\dagger}$ Senior Research Scientist, Aeroacoustics Branch, MS 461, Fellow AIAA.

* Senior Research Engineer, Aeroacoustics Branch, MS 461, Senior Member AIAA.

$\S$ Senior Engineer, Lockheed Martin Space Operations, Langley Program Office.
} 


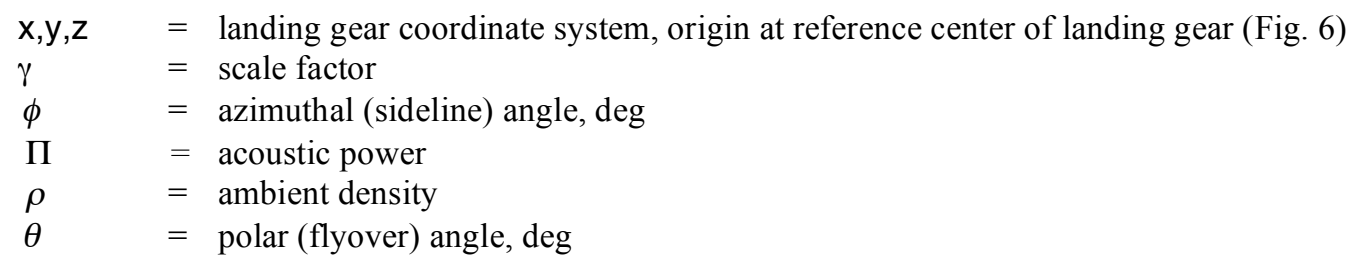

$\begin{array}{ll}\text { Subscripts } & \\ e & =\text { emission } \\ f S & =\text { full-scale } \\ H & =\text { high frequency component (Guo method) } \\ L & =\text { low frequency component (Guo method) } \\ M & =\text { mid frequency component (Guo method) } \\ m s & =\text { model-scale }\end{array}$

\section{Introduction}

Aircraft system noise prediction capabilities are necessary to estimate the community noise impact of future aircraft as well as provide noise impact estimates of changes in propulsion systems, airframes, configurations, and flight operations of current aircraft. Aircraft system noise is the cumulative sum of noise generated by various components of the aircraft propulsion system, various components of the airframe (including the landing gear) and their interaction effects. Landing gear are a prominent source of airframe noise, which is the focus of this paper. Predicting noise on the ground from an aircraft flyover requires estimating the noise generated by the many contributing sources during the flyover as the flight conditions change, summing these sources as a function of time, and propagating the resultant combined sources through the atmosphere to the observer locations. NASA introduced the Aircraft NOise Prediction Program ${ }^{1,2}$ (ANOPP) about 30 years ago to provide such a capability for predicting the noise from aircraft in flight. NASA has continued to upgrade, add and improve these prediction capabilities through validation and advanced modeling developments. The ANOPP system is used by both government agencies and the aircraft industry to assess existing aircraft as well as evaluate the noise reduction potential of new technologies. This requires that the prediction models of ANOPP be computationally efficient, yet of sufficiently high fidelity to capture the effects on noise of design and/or operational changes. Hence, ANOPP relies primarily on semi-empirical methods for predicting the various aircraft noise sources.

In recent years, with the introduction of higher bypass ratio engines and quiet engine technologies, the noise from the propulsion system has been reduced on approach such that airframe noise and engine noise are of comparable levels. The airframe noise components that dominate during approach depend on the configuration, but are typically found to be those associated with the landing gear and/or the high-lift assemblies, such as the flaps, slats and ailerons. The prediction of these sources, in particular those associated with the landing gear, has been of interest for a number of years. Early studies of landing gear noise date back to the 1970's with the work of Heller and Dobrzynski ${ }^{3}$ who investigated moderately complex two- and four-wheel geometries, yielding separate model scale and flight scale data that could be compared for the first time. During this time frame Fink ${ }^{4,5}$ created empirical formulations that were calibrated with the data of Heller and Dobrzynski. This empirical methodology was implemented in ANOPP and provided the capability to predict one-third-octave noise spectra based on a few fundamental parameters such as: mean flow velocity, number of wheels, wheel diameter and landing gear strut length. The validation and further development of a higher fidelity model was somewhat hindered due to the scarcity of measurements from flight and wind tunnels.

Interest in landing gear noise subsided in the 1980's, but has gained increased interest since the late 1990's with the experimental work of Dobrzynski et al. ${ }^{6,7,8}$, Michel et al. ${ }^{9}$, Jaeger, et al. ${ }^{10}$, Horne et al. ${ }^{11}$, Stoker et al. ${ }^{12}$, Humphreys et al. ${ }^{13}$ and the advanced modeling work of Smith et al. ${ }^{14}$, Molin et al. ${ }^{15}$, Guo ${ }^{16,17,18}$ and computational aeraocoustic methods of Hedges et al. ${ }^{19}$, Seror et al. ${ }^{20}$, and Lockard et al. ${ }^{21}$ The experimental work has provided 
much needed data to advance the understanding of the noise generation mechanisms and to characterize the directivity of the radiated noise leading to the improvement and validation of prediction capabilities. The computational aeroacoustic methods are still too inefficient (although much progress has been made) to be included in a system capability such as ANOPP. However, the newer, although still semi-empirical, landing gear models of $\mathrm{Guo}^{16,17,18}$ are appropriate and have been shown to compare well with measured full-scale data obtained in flight and wind tunnel testing ${ }^{17}$. The prediction models of Guo have been implemented within ANOPP as they become available. The most recent improvements include the capability to calculate the local flow velocities for gears installed on aircraft in flight, modeling for the noise generated by the nose gear, and the far-field directivity for all angles. ${ }^{18}$

The main focus of the present paper is to assess and compare ANOPP landing gear noise prediction methods with measured model-scale data obtained from a recent experimental study performed by Humphreys and Brooks ${ }^{13}$ in the NASA Langley Quiet Flow Facility (QFF). This detailed study involved the acquisition of aeroacoustic data from a high-fidelity, 6.3\%-scale Boeing 777 main gear with an emphasis on determining hemispherical spectral and directivity levels as well as verifying velocity scaling laws. The ANOPP methods are examined for their ability to predict noise for a small-scale landing gear model. Predictions are made using both methods implemented in ANOPP and compared with measured spectra at different directivity angles (polar and azimuthal). In addition the ability of the prediction methods to capture the noise directivity is examined by comparing predicted noise contours with measurement. These comparisons provide an opportunity to assess and modify the directivity characteristics of the ANOPP prediction methods, which were implemented based on limited data.

\section{ANOPP Landing Gear Noise Prediction Methods}

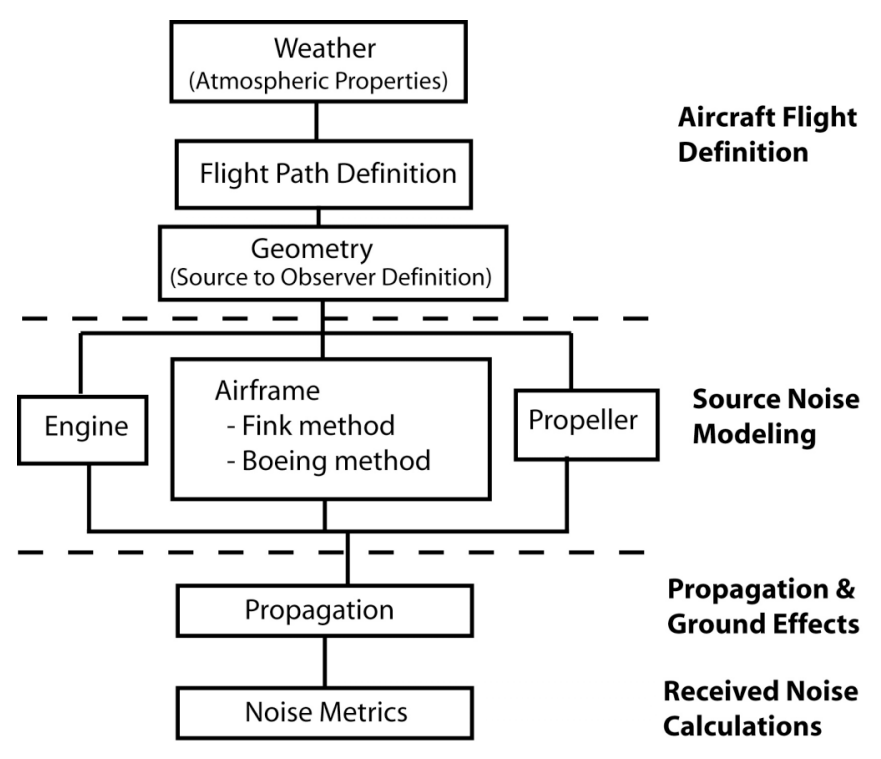

Figure 1. Schematic of the subsonic aircraft analysis part of the Aircraft System NOise Prediction Program (ANOPP).

A schematic of the ANOPP system is shown in Fig. 1. ANOPP consists of a set of functional modules that predict atmospheric properties, aircraft flight path, source-to-observer geometry, source noise of the aircraft components (propulsion systems and airframe), propagation of the source noise to ground observers, and community noise metrics. Not shown, but an important part of the capability is the ANOPP executive. It not only controls the execution of modules but interprets and creates inputs for modules, provides data management for the functional modules and provides error handling and exit procedures to the host computer.

Within the suite of ANOPP source noise modules, two methods are available for predicting airframe noise. One method was developed by Fink, ${ }^{4,5}$ the other by Sen et al ${ }^{22}$, which has been improved by Guo ${ }^{16,17,18}$ in recent years. Both methods predict one-third octave band noise for the wing and landing gear components. For this paper, the focus is on the landing gear noise prediction.

While most often used to predict the noise for a vehicle in flight, ANOPP can be used to study an individual noise source such as the landing gear. In this mode, the source noise is output to a spherical array of stationary observers located around the source. The noise at these observers includes source motion effects such as the Doppler 
frequency shift and convective amplification. To compare the ANOPP predicted noise levels with wind tunnel data; the output was modified by removing only the Doppler frequency shift. The formulations presented in the next section reflect this modification.

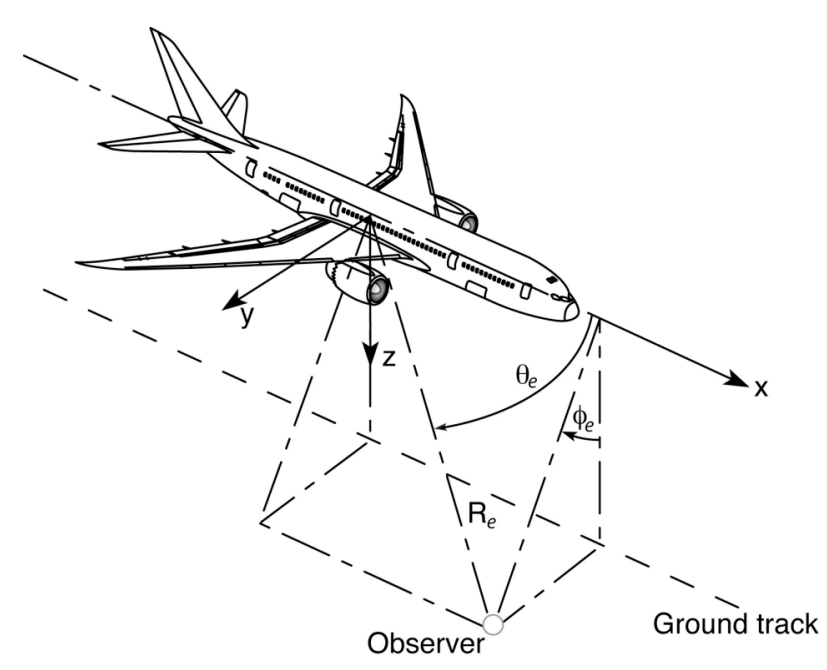

Figure 2. Coordinate system used in ANOPP.

\section{A. ANOPP Fink Method}

Fink developed the landing gear noise element of his prediction method by fitting equations to data for two-wheel and four-wheel landing gear configurations. $^{4,5}$ Correlations were developed based on the assumption that landing gear noise can be expressed in terms of the major gear components. Therefore the inputs to the model are simple and require only the number of wheels, the wheel diameter and the length of the main strut to be specified. Separate equations were developed for the main gear and nose gear. For either landing gear type (main or nose), the noise is assumed to be generated from the flow interactions with and within the wheel assemblies and the main strut. Separate predictions are made for the strut and the wheel noise, which are subsequently summed on a p-squared basis to yield the total landing gear noise.

The general form of the ANOPP far-field mean-square acoustic pressure formulation of the Fink landing gear method is

$$
\left\langle p^{2}\right\rangle=\frac{\rho c \Pi}{4 \pi R_{e}^{2}} \frac{D\left(\theta_{e}, \phi_{e}\right) F\left(S_{t}\right)}{\left(1-M \cos \theta_{e}\right)^{4}}
$$

where $\Pi$ is the acoustic power, and $D$ is the directivity function. $F$ is the spectrum function, and $R_{e}$ is the source-toobserver distance. $M$ is the aircraft Mach number and $\left(1-M \cos \theta_{e}\right)^{4}$ is the convective amplification factor.

The wheel noise acoustic power is defined by

$$
\Pi_{\text {wheel }}=K_{1} M^{6} \mathrm{~N}_{\mathrm{w}} d^{2}
$$

where $K_{1}=4.349 \times 10^{4}$ for one or two-wheel landing gear, and $K_{1}=3.414 \times 10^{4}$ for four-wheel landing gear. The strut noise acoustic power is defined by

$$
\Pi_{\text {strut }}=K_{2} M^{6} d L
$$

where $\mathrm{K}_{2}=2.753 \mathrm{E}-4$. The strut and wheel contributions are of similar order of magnitude and would be nearly equal if $L=\mathrm{N}_{\mathrm{w}} d$. For the Boeing 777 main gear, the strut length is approximately 3 times the wheel diameter. The noise directivity of the strut is significantly different than that of the wheel assembly. The directivity function for the noise generated by the wheel assembly is

$$
D\left(\theta_{e}, \phi_{e}\right)=\frac{3}{2} \sin ^{2} \theta_{e}
$$


The directivity function for the noise generated by the strut is

$$
D\left(\theta_{e}, \phi_{e}\right)=3 \sin ^{2} \theta_{e} \sin ^{2} \phi_{e}
$$

Note that when $\phi_{e}=0^{\circ}$, the strut directivity function is zero, and hence the contribution from the strut does not contribute to the overall noise level directly under the landing gear. This will become more evident when the total and component predictions are compared with measured spectra.

The spectrum functions $F$ for the wheel and strut are defined as a function of the Strouhal number $S_{t}$ in the following form

$$
F\left(S_{t}\right)=A \frac{S_{t}^{n}}{\left(B+C S_{t}^{m}\right)^{q}}
$$

where $A, B, C, n, m$ and $q$ are empirical constants uniquely defined for the strut and the wheel ${ }^{1,5}$. The Strouhal number for both the strut and wheel spectrum functions is defined using the wheel diameter as the characteristic length scale,

$$
S_{t}=\frac{f d}{M c}
$$

It should be noted that for landing gear with more than 4 wheels, the prediction method defaults to the four-wheel formulation. This is a limitation of the method. In the results section, predictions are made using both two- and fourwheel formulations and presented for comparison.

\section{B. ANOPP Guo Method}

The Guo method is based on fundamental aerodynamic noise theory, statistical scaling laws derived from the theory, and correlations of these laws with full-scale landing gear noise tests ${ }^{16}$. The method decomposes the landing gear noise into three spectral components. The low frequency spectral component represents the noise generated by large-scale structures such as wheels. The mid and high frequency spectral components correspond to the main struts and the small-scale details such as hydraulic lines respectively. The spectral shape, amplitude, and directivity of each component were derived separately, and each contains factors to allow modeling of the details of each spectral component in an attempt to include more physics. The Guo method requires significantly more detailed inputs to define the landing gear assembly than the Fink method, hence, providing a higher fidelity prediction capability. These inputs include all the basic flow and geometric parameters used in the Fink method: i.e., number of wheels; wheel diameter; main strut length; but also the wheel width, axle diameters, bogie length and width, as well as details on all other struts, shock struts, vertical bars, axles, torque bars, junction rods and door details. In addition and importantly, unlike the Fink method, the Guo method was developed in terms of a narrowband spectrum, which is converted to one-third-octave as the last step. This has ramifications regarding frequency scaling as will be indicated later. The general form of the far-field mean-square acoustic pressure formulation is

$$
\left\langle p^{2}\right\rangle=\frac{\rho^{2} c^{4} M^{6} D_{0}\left(\theta_{e}\right)}{R_{e}^{2}\left(1-M \cos \left(\theta_{e}\right)\right)^{4}}\left\{P_{L}+P_{M}+P_{H}\right\}
$$

where the parameters common to all three spectral components have been factored out, and those specific to a particular component are represented by the $P$ terms. The subscripts $L, M$ and $H$ denote the low, mid and high frequency components, respectively. The overall directivity $D_{0}\left(\theta_{e}\right)$ is in addition to the directivity function defined within each $P$ term and is included to account for the effects of the landing gear installation. To simulate wind tunnel 
conditions, the factor $\left(1-M \cos \theta_{e}\right)^{4}$ should have been included to account for convective amplification, but was erroneously excluded from the method. ${ }^{23}$ The factor has been re-inserted for the predictions of this paper. Each $P$ takes on the form

$$
P=\beta \mathrm{S} D\left(\theta_{e}, \phi_{e}\right) F\left(S_{t}\right)
$$

where $\beta$ is the flow energy conversion efficiency parameter, which models the efficiency of the process whereby the steady flow motion of the landing gear parts generates unsteady flow that radiate noise. Currently, this parameter can only be determined by matching prediction with data. $\mathrm{S}$ is the aggregate surface integration effects parameter and is related to the aggregate surface area of the landing-gear parts for each frequency component. For the low frequency component it is computed directly from the wheel dimensions. For the mid frequency component it is computed by summing the surface areas of all main strut elements. For the high frequency component that approach is not practical because of the large number of small parts, which have various shapes and orientations. Hence, $S_{H}$ is modeled using an empirically defined length scale and 'complexity factor', $\eta$, which has the following form for main gears

$$
\eta=1+0.15\left(\frac{W}{W_{\text {ref }}} \frac{L_{L}}{L_{L_{\text {ref }}}} \frac{N_{w}}{N_{w_{\text {ref }}}}-1\right)
$$

The $W, L_{L}$, and $N_{w}$ terms are the maximum takeoff gross weight of the aircraft, the total length of all the main struts and the number of wheels, respectively. The subscript ref indicates reference values, determined from full-scale dat $^{18}$. The functions $\mathrm{D}$ and $\mathrm{F}$ are the component specific directivity and spectrum functions, respectively. The general form of the directivity function is

$$
D\left(\theta_{e}, \phi_{e}\right)=\left(1+h \cos ^{2} \theta_{e}\right)^{2}\left(1+b \sin ^{2} \phi_{e}\right)
$$

where $h$ and $b$ are unique for each of the three frequency domains. The values for the low, mid, and high frequency domains respectively are $h=(0.2,0.6,1.0)$ and $b=(3.0,2.0,0.1) .{ }^{18}$ The spectrum function $F$ is a narrowband power spectral density function and has the following form

$$
F\left(S_{t}\right)=A \frac{S_{t}^{\sigma}}{\left(B+S_{t}^{\mu}\right)^{q}}
$$

where $\sigma, \mu$ and $\mathrm{q}$ are empirical constants which define the spectral shape; A, and $\mathrm{B}$ are derived constants that set the peak value to unity at an empirically derived Strouhal number $S_{t 0}$. For each of the three frequency component spectra, a unique set of $\sigma, \mu, \mathrm{q}, \mathrm{A}, \mathrm{B}$ and $S_{t 0}$ was determined by analyzing full-scale test data. ${ }^{17}$ The Strouhal number $S_{t}$ is determined using the characteristic length scale associated with the frequency range for which $F$ is being evaluated. The characteristic length scale for the low frequency component spectrum is the wheel diameter; whereas, the characteristic length scale for the mid and high frequency component spectra are computed using the aggregate surface area parameter (S), an average length, and an empirical scale factor. Complete details on the development of these factors and parameters are fully documented in Refs. 17 and 18.

Since $F$ is defined as a narrowband spectral function, its shape is not invariant with $S_{t}$, which undercuts the generality of using Strouhal scaling on which it is based. This effect, combined with the empirical function $\eta$ used to define $\mathrm{S}_{\mathrm{H}}$, makes the Guo method, at present, only appropriate for full-scale landing gear. However, the model does attempt to incorporate as much physics as possible to reduce configuration dependence, but it does not eliminate empiricism. It should be noted that this method compares very well with full-scale landing gear measurements ${ }^{16,17}$ and is considered an improvement over existing semi-empirical prediction capabilities for full-scale 777 and 737 gear predictions.

For this paper, the inputs to both prediction methods utilized the geometric description of the full-scale Boeing 777 main gear provided in Ref. 18 and given here in Tables 1 and 2 for completeness. As mentioned earlier, the Fink 
method only requires as input the number of wheels and their diameter, the length of the main strut, and the free stream Mach number. The Guo method allows for many more geometric details. The entire list specified in Tables 1 and 2 were utilized in the Guo predictions of this paper. The Fink method used scaled dimensions as will be explained in the Results and Discussion section.

Table 1 - General Input Parameters (full-scale) from Ref. 18

\begin{tabular}{|l|c|}
\hline Input Parameters & Value \\
\hline Free Stream Mach Number & 0.17 \\
\hline Source-to-Observer Distance (ft) & 79.365 \\
\hline Angle-of-Attack (deg) & 0.0 \\
\hline Wheel Track Angle (deg) & 13.0 \\
\hline Number of Tires & 6 \\
\hline Tire Diameter (in) & 50.0 \\
\hline Tire Width (in) & 20.0 \\
\hline
\end{tabular}

Table 2 - Main Gear Component Dimensions (full-scale) from Ref. 18

\begin{tabular}{|l|c|c|c|}
\hline Main Gear Components & $\begin{array}{c}\text { Length } \\
\text { (in) }\end{array}$ & $\begin{array}{c}\text { Diameter / } \\
\text { Width (in) }\end{array}$ & Thickness (in) \\
\hline Shock Strut & 153.0 & 16.0 & - \\
\hline Upper Hydraulic Rod & 30.0 & 12.0 & - \\
\hline Lower Hydraulic Rod & 35.0 & 3.0 & - \\
\hline Axle & 105.0 & 8.5 & - \\
\hline Axle Connection & 120.0 & 13.0 & - \\
\hline Front Hydraulic Rod & 54.0 & 7.6 & - \\
\hline Lower Front Side Bar & 50.0 & 7.0 & 6.0 \\
\hline Lower Aft Side Bar & 50.0 & 7.0 & 6.0 \\
\hline Upper Front Side Bar & 54.0 & 8.0 & 6.0 \\
\hline Upper Aft Side Bar & 54.0 & 8.0 & 6.0 \\
\hline Horizontal Front Side Bar & 48.0 & 4.0 & 4.0 \\
\hline Horizontal Aft Side Bar & 43.0 & 4.0 & 4.0 \\
\hline Upper Torque Bar & 58.0 & 5.0 & 3.0 \\
\hline Lower Torque Bar & 64.0 & 5.0 & 3.0 \\
\hline Rear Wheel Steering & 25.0 & 2.5 & - \\
\hline Rear Wheel Hydraulic & 20.0 & 5.0 & - \\
\hline
\end{tabular}

\section{QFF Landing Gear Test}

\section{A. Test Description and Data Acquisition}

A high-fidelity 6.3\%-scale model of a Boeing 777 main landing gear assembly was recently tested in the NASA Langley Quiet Flow Facility (QFF) ${ }^{13}$. This high complexity gear model, shown in Fig. 3, is comprised of a six-wheel bogie and a generally faithful representation of full-scale strut, oleo (main shock strut), braking and hydraulic hardware. The dimensions of this test model were verified to be $6.3 \%$ of the dimensions provided in Table 1 for the full-scale Boeing 777 landing gear. More complete details of this model can be found in Ref. 13. However, it should be noted that the hydraulic lines and cables dimensions of the scale-model were generally slightly larger than $6.3 \%$ of full-scale. This may be expected to have the effect of shifting the high frequency content of the scale-model noise down slightly when compared with noise measured from actual full-scale gear. 


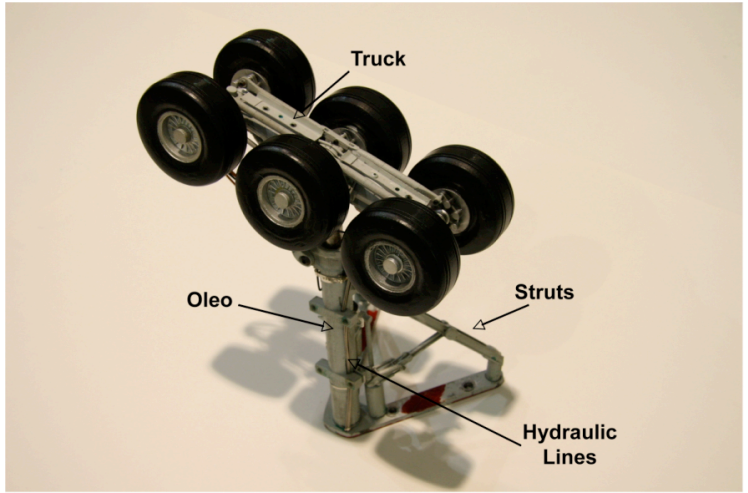

a. Main landing gear shown without the door.

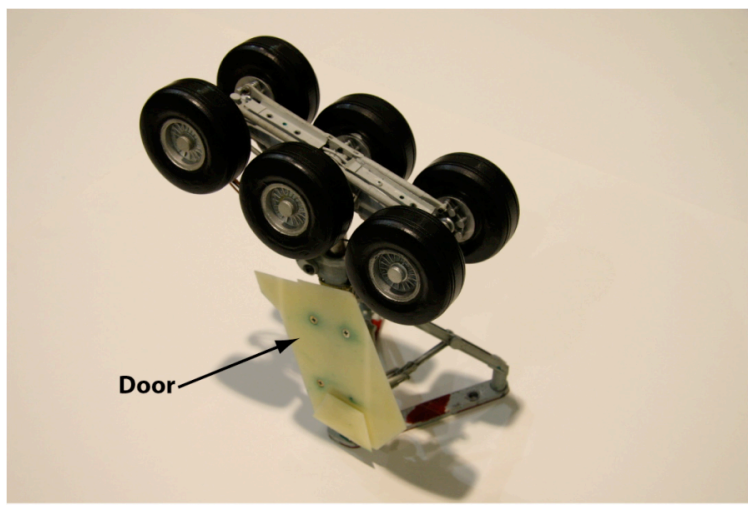

b. Main landing gear baseline configuration (with the door).

Figure 3. "High-Fidelity," Boeing 777, 6.3\% scale-model main landing gear assembly.

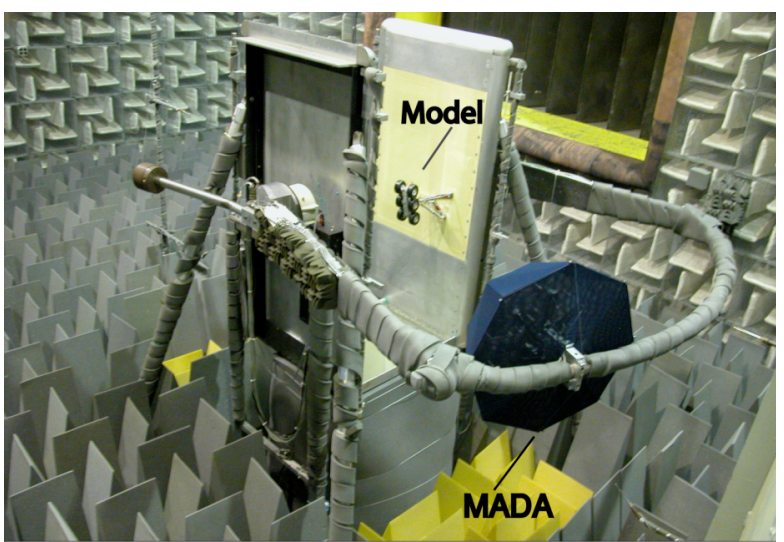

Figure 4. Model mounted in QFF for "sideline" viewing.

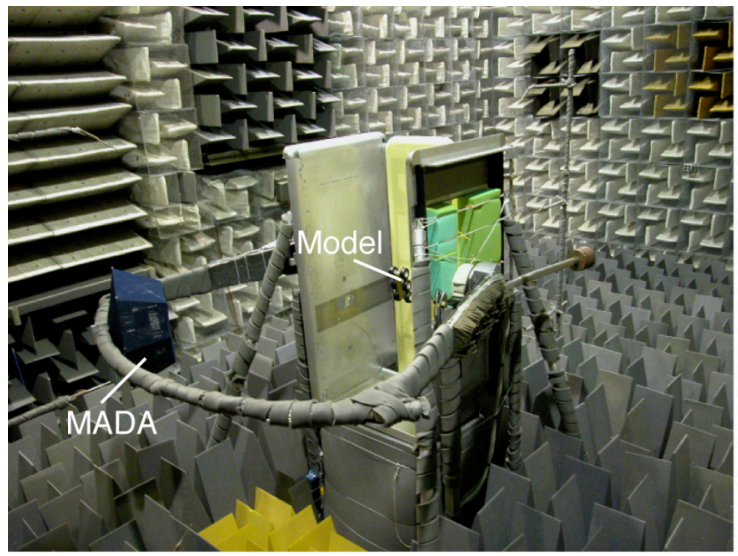

Figure 5. Model mounted in QFF for "flyover" viewing.

The Quiet Flow Facility (QFF) is an open-jet anechoic facility specifically designed for aeroacoustic testing. The shorter sides of the 2 by 3 foot nozzle are bounded by fixed sideplates to which test articles can be attached. The model configuration shown in Fig. 4 was used to obtain "sideline" viewing of the landing gear by the boommounted microphone array called MADA (Medium Aperture Directional Array ${ }^{13}$ ). For "flyover" viewing of the landing gear, the model was mounted on a specially constructed wall that was inserted on an adjacent wall for these measurements as shown in Fig. 5. The angle of the truck with respect to the free stream was 13 degrees.

For either orientation of the landing gear model, the coordinate system shown in Fig. 6 was employed during analysis of the directivity measurements. The reference center of the landing gear is defined to be the center of the truck assembly. This coordinate system, defining the $\mathrm{x}$-axis as being aligned with the direction of flight and the $\mathrm{z}$ axis directed under the flight path, corresponds to the coordinate system used in ANOPP as shown in Fig. 2. Using this coordinate system, observer locations were specified by an observer distance $R_{e}$ and two angles, with $\theta_{e}$ denoting the polar flyover angle and $\phi_{e}$ denoting the azimuthal sideline angle. Reference 13 reports on an extensive analysis used to derive equivalent emission coordinates in order to provide measurements in a coordinate system that can be used for free-field correlations. This requires not only coordinate system considerations, but also proper application of shear-layer acoustic refraction corrections critical to obtaining accurate amplitudes. 


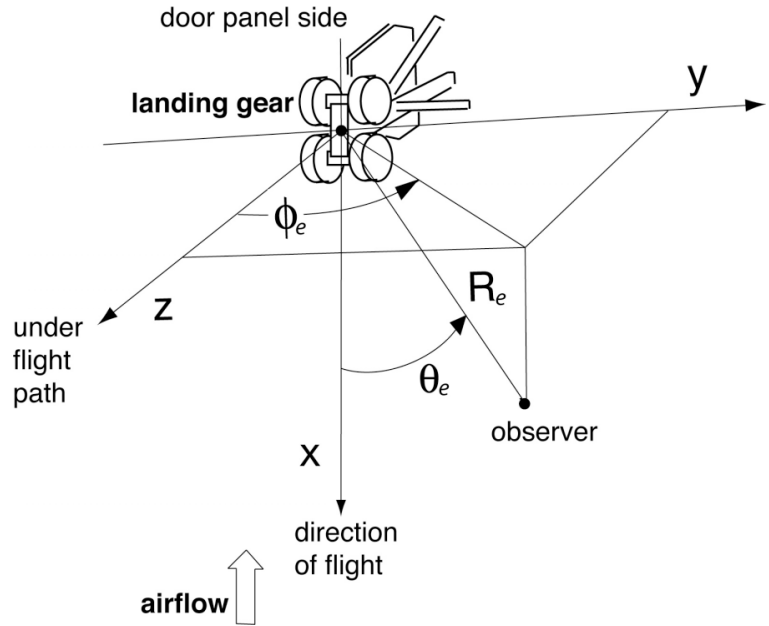

Figure 6. Quiet flow facility coordinate system for landing gear.

Acoustic spectra and directivity of landing gear components were obtained with the Langley Medium Aperture Directional Array (MADA). The MADA consists of $41 \quad$ B\&K $4138 \quad 1 / 8$-inch microphones with 1/4-inch preamplifiers mounted on an acoustically treated frame and covered with a hood of thin, woven nylon fabric to reduce microphone self-noise due to recirculating air currents within the QFF. The entire assembly was mounted on a pivoting boom, designed to allow positioning of the array at a number of polar and azimuthal angles, as shown in Figs. 5 and 6. The MADA has five circles of eight microphones each, and a microphone in the center. The measured results of this paper are from four single microphones located on the outer circle of the MADA, which is nominally $5 \mathrm{ft}$. from the center of the landing gear. The measured data were processed to form autospectra, which were then corrected for shear layer refraction. All data presented in this paper are in asmeasured model-scale frequencies, and the amplitudes are referenced to a five-foot distance between the model and microphones. Details of this processing are found in Ref. 13.

\section{B. Test Matrix}

Table 3 is a summary of the test matrix for the baseline model study. The baseline landing gear model, which includes the door, was tested for a Mach number range of 0.11 to 0.17 to simulate typical approach velocities for the Boeing 777 aircraft. It should be noted that the two prediction methods in ANOPP were developed using data from configurations that also included the door. Finally, a range of polar and azimuthal angles was chosen for acquisition such that a sizeable portion of the hemispherical noise directivity of the gear could be obtained. The measured results are presented in the form of autospectra and acoustic directivity maps of specific one-third-octave band sound pressure levels (SPL, dB). Complete details of the data acquisition, microphone locations, and data processing are documented in Ref. 13.

Table 3 - Test Matrix Summary of Baseline Model Study

\begin{tabular}{|l|l|}
\hline Model Configuration & $\begin{array}{l}\text { High-Fidelity B777 baseline model } \\
\text { (includes door) }\end{array}$ \\
\hline Model Mounting / Views & Sideline, Flyover \\
\hline Mach Numbers & $0.11,0.13,0.15,0.17$ \\
\hline Reynold's number range (based on wheel diameter) & Approx. $2.1 \times 10^{5}$ to $3.3 \times 10^{5}$ \\
\hline 'Polar Angles' (emission angles) & Nominal range: 55 to 135 degrees \\
\hline 'Azimuth Angles' (emission angles) & Nominal range: -106 to 94 degrees \\
\hline
\end{tabular}




\section{Results and Discussion}

In the following sections predicted results from the two ANOPP methods are presented in the form of one-thirdoctave spectra and noise directivity maps that are compared to measurement. Before this is presented, the validity and applicability of each method to predict model-scale landing gear noise is addressed. In addition, because each method has options for modeling and execution, these are discussed, and the rationale for selecting specific options is explained. The comparison of each method to measured spectra and noise directivity is then presented. It should be noted that the predicted and measured one-third-octave spectra are presented as solid lines with and without symbols, even though the amplitudes were only actually defined at discrete one-third-octave center band frequencies.

\section{A. Full-Scale Prediction and Model-Scale Data Comparison Method}

Both the Fink and Guo prediction methods were developed using full-scale landing gear noise data to define the noise spectral shape and amplitude. The Fink method is a pure Strouhal scaling approach that allows prediction of any landing gear size. The Guo method is also based in large part on the Strouhal scaling approach. However, as mentioned in relation to Eq. (10) the employment of "complexity factors" to produce agreement to full-scale landing gear data along with a narrowband (versus one-third-octave-band) spectrum function prevents the prediction of arbitrary landing gear sizes. In this section, the scaling behavior of the present data and the predictions are examined and a method is "Derived" to correctly compare full-scale predictions with model data.

Traditional Strouhal spectral scaling assumes both geometric and flow similarity for a noise producing body in the flow. Define in this example $S_{t}=f \ell / U$, where $\ell$ can be any body length-scale (such as, $d$ in the Fink method). Noise produced over particular $S_{t}$ values are assumed to be caused by similarity-related turbulent-flow structures (eddies) with respect to the body, independent of particular $f$, $\ell$, or $U$ values. One-third octave spectra are a natural format for scaling and prediction because the energy distribution in the frequency bands is proportional to frequency and, thus, Strouhal number, $S_{t}$. Strouhal spectral scaling can be represented by

$$
S P L_{1 / 3}-10 \log \left[M^{6}\left(\ell / R_{e}\right)^{2}\right]=F\left(S_{t}\right)+K
$$

Here, for a particular landing gear configuration and observer direction, $F\left(S_{t}\right)$ has a set function shape, and $K$ is a constant. (If the $S P L$ in Eq. (13) were in terms of a constant bandwidth, an invariant form for $F\left(S_{t}\right)$ would not be possible. Its bandwidth $\Delta S_{t}$ would have to depend on $\ell$ and/or $U$ values.) Equation (13) is similar in form to Eq. (19) in Ref. 24, except that, here, a sixth power (appropriate for blunt body flow noise) is used for Mach number dependence rather than the fifth power (appropriate for boundary-layer-turbulence trailing edge noise). Equation (13) is not applied here to the data directly, but it is consistent with prediction elements in the Fink method. It is used to help establish proper comparisons with the Guo's method.

Taking Eq. (13) to be generally applicable, with matched Mach numbers, the $S P L_{1 / 3}$ spectrum shape and level would be identical for model-scale landing gears with representative length scales of $\ell_{m s}$ and full-scale landing gears with $\ell_{f s}$ as long as

$$
\left(\ell / R_{e}\right)_{m s}=\left(\ell / R_{e}\right)_{f s}
$$

and

$$
\left(S_{t}\right)_{m s}=(f \ell / U)_{m s}=\left(S_{t}\right)_{f s}=(f \ell / U)_{f s},
$$

so

$$
f_{m s}=\left(\ell_{f s} / \ell_{m s}\right) f_{f s}=(1 / \gamma) f_{f s}
$$


and

$$
\Delta f_{m s}=\left(\ell_{f s} / \ell_{m s}\right) \Delta f_{f s}=(1 / \gamma) \Delta f_{f s}
$$

These give the basis for full-scale predictions with model-scale data. In Eq. (15), $\gamma$ is the model scale factor 0.063 and thus $f_{m s}=15.9 f_{f s}$ and $\Delta f_{m s}=15.9 \Delta f_{f s}$. No level adjustment is needed as long as $\left(R_{e}\right)_{f s}=(1 / \gamma)\left(R_{e}\right)_{m s}$ from Eqs. (14).

Note that Eqs. (14)-(16) do not exclusively depend on Strouhal scaling as shown in Eq. (13). These equations can also follow from an acoustic scaling approach detailed by Schmitz et al. ${ }^{25}$ and employed by Brooks et al. ${ }^{26}$ for model- to full-size rotorcraft noise scaling. For flow similarity, normalizing the Ffowcs-Williams and Hawkings equation geometrically and temporally for matched Mach numbers and normalized pressure coefficients also produces like similarity in acoustic time histories and spectra.

Figure 7 shows three predicted one-third-octave spectra (no experimental data is shown). The solid blue line is a prediction using the Fink method for the present landing gear model for an observer at emission polar and azimuth angles of $\theta_{e}=87.1^{\circ}$ and $\phi_{e}=-1.0^{\circ}$ at $R_{e}=5 \mathrm{ft}$. Equation (13), along with Eqs. (14) and (15), indicate that the same spectrum should be attainable by predicting the spectrum of a full-scale landing gear at the same observer polar and azimuthal observer angles but at a $R_{e}$ of 15.9 x $5 \mathrm{ft}=79.5 \mathrm{ft}$, although the frequencies would be shifted lower by a factor of 0.063 . This full-scale landing gear predicted spectrum is shown by the open symbols in Fig. 7 . The fullscale prediction spectrum is shifted in frequency using Eq. (15) to obtain the spectrum with closed symbols. This shows exact agreement with the model prediction. In this paper, this shifted spectrum is termed a 'Derived' model scale prediction.

For the same conditions used in Fig. 7, Fig. 8 shows the corresponding predicted spectra from the Guo method. The full-scale model prediction is seen to have a somewhat similar shape and level as that of Fink. This should not be surprising since Guo and Fink both employed full-scale landing gear data for their predictive modeling. The Guo method prediction of the present test model is shown by the solid line. It is seen to have a much higher level and a frequency shift different from than shown in Fig. 7 for the Fink prediction. However, when one uses the present 'Derived' model scale method on the Guo full-scale prediction, a much closer match to the Fink model scale prediction is seen. For the remainder of the paper, Fink predictions are made at model scale. The Guo predictions are based on full-scale input and adjusted to equivalent test model scale using the 'Derived' method of this paper. In many respects this can be viewed as a comparison of test model data to that of full-scale data to the extent that the Fink and Guo predictions represent accurate descriptions of full-scale spectra.

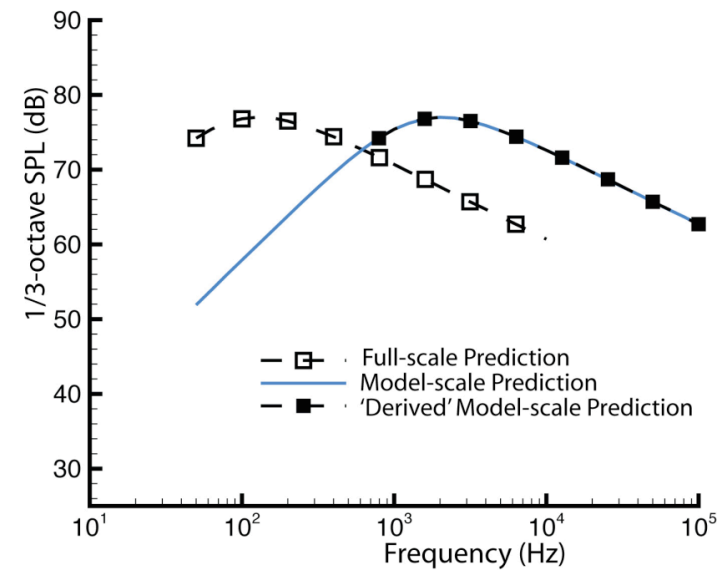

Figure 7. Predicted spectra using the Fink method for an observer position at $\theta_{e}=87.1^{\circ}, \phi_{e}=-1.0^{\circ}$.

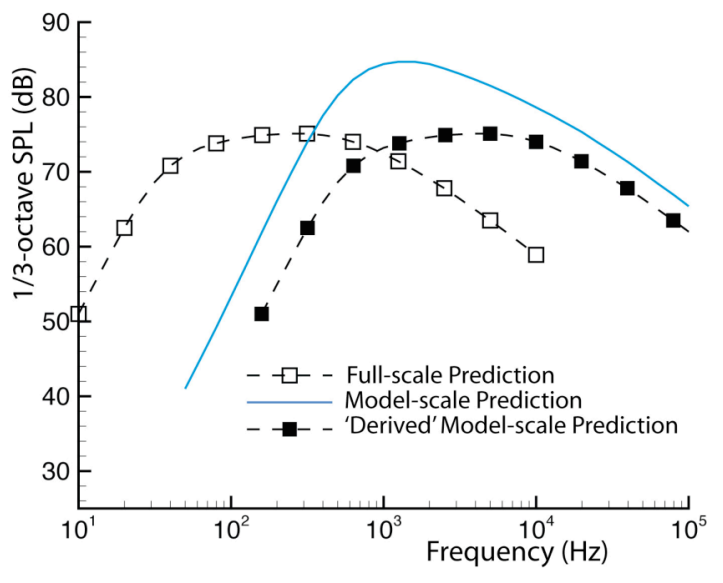

Figure 8. Predicted spectra using the Guo method for an observer position at $\theta_{e}=87.1^{\circ}, \phi_{e}=-1.0^{\circ}$. 


\section{B. Predictions and Test Model Comparisons}

Both prediction methods examined in this report, as well as Eq. (13), employ a velocity to the $6^{\text {th }}$ power dependence. Reference 13 presents one-third octave presentations of spectra for the flyover position for the baseline model for a range of tunnel speeds. These spectra are scaled, in a manner consistent with Strouhal scaling, in Fig. 9 using the $M=0.17$ data to specify the reference amplitude and frequency. Initial level differences of $16 \mathrm{~dB}$ (without normalization), are reduced to the several-dB range shown with the normalization. Similar scaling success using the $6^{\text {th }}$ power was found over a range of directivities examined in the study of Ref. 13. For clarity of presentation, no prediction comparisons are made in Fig. 9. For the remainder of this report, only Mach number $M=0.17$ cases are examined with regard to spectra and directivity. The measured data include atmospheric attenuation, which is less than $0.5 \mathrm{~dB}$ below $12.5 \mathrm{kHz}$ and on the order of $1 \mathrm{~dB}$ at $40 \mathrm{kHz}$ for the ambient conditions tested. The predictions do not include atmospheric attenuation.

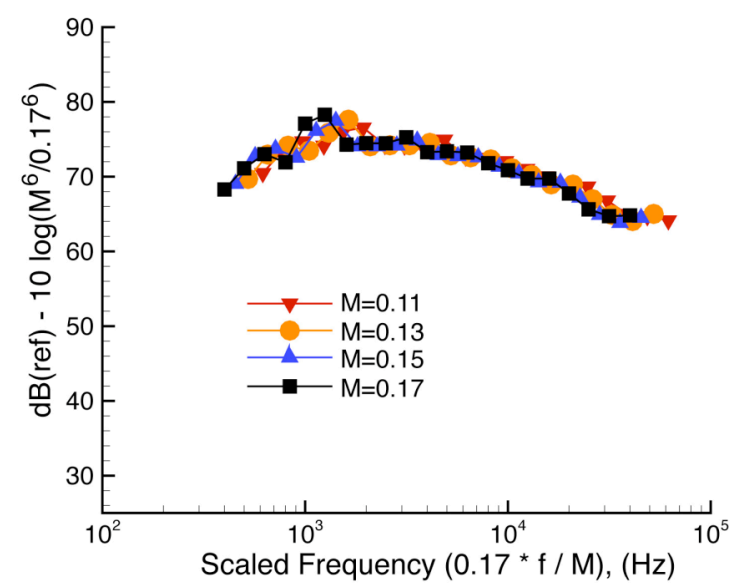

Figure 9 Normalized QFF model landing-gear spectra for a range of test Mach numbers. Observer at $\theta_{e}=87.1^{\circ}, \phi_{e}=-1.0^{\circ}$, and $R_{e}=5 \mathrm{ft}$.
As mentioned earlier the Fink method is based on empirical formulations validated for two-wheel and four-wheel landing gear configurations. The Boeing 777 scale-model landing gear has 6 wheels. For landing gear configurations with more than 4 wheels, the prediction method defaults to the four-wheel formulation. In Fig. 10, Fink method predictions are compared to measured data obtained at a flyover observer location. The two-wheel prediction shows fair comparison with the measured results. The four-wheel prediction (presented also in Fig. 7) compares surprisingly well to the measurement. For the remainder of the paper, the Fink predictions are based on the fourwheel formulation, realizing that the measured data are from the six-wheel Boeing 777 main gear model.

The full-scale dimensions of the Boeing 777 main landing gear used in the Guo method are provided in Tables 1 and 2. The Guo method offers two options for predicting noise from the main gear. One option is known as "detailed geometry" the other as "estimated geometry". The detailed geometry option, where the dimensions of each strut component that contributes to the mid-frequency component spectrum are specified, is the option developed by Guo for his full-scale landing gear prediction. The "Derived" prediction corresponding to this option is shown in Fig. 11 (presented also in Fig. 8) along with measurements from the QFF model-scale test. It is seen that reasonably good comparison is made.

For completeness and comparison, a "Derived" prediction using the "estimated geometry" option is also shown in Fig. 11. This option is proposed by Guo when it is not be practical to obtain detailed dimensions for particular gears or when only a more approximate estimate of noise is needed. For these predictions, only main component length scales are input. The comparison shown in Fig. 11 shows less agreement with the present scale model data than that of the "detailed geometry" prediction.

Again for completeness, predictions were also made using the geometric inputs used for the main gear, but specifying a nose gear formulation. The nose gear formulation is similar to that for the main gear, but employs a substantially different installation directivity function $\left(D_{0}\right)$, flow energy conversion efficiency $(\beta)$ and complexity factor $(\eta)$. Comparison using this option was suggested by Guo. ${ }^{18}$ The rationale would be that scale-model gears tend to be geometrically simple compared to their full-scale counterparts, they would possibly be more similar to aircraft nose gear than main gear. The "Derived" predictions using the nose gear mode, with both the detailed and estimated geometry options are shown in Fig. 11. These are seen to under predict the measured data by at least $5 \mathrm{~dB}$ for all frequencies. For the remainder of the paper, the "detailed geometry" option is used to compute the full-scale landing gear from which the "Derived" scale-model predictions are obtained.

12

American Institute of Aeronautics and Astronautics 


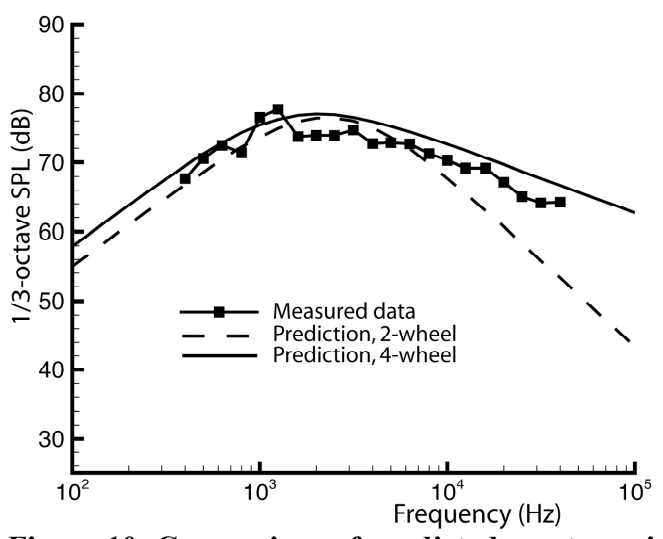

Figure 10. Comparison of predicted spectra using the Fink method for a 2-wheel and 4-wheel landing gear assembly, $\left(\theta_{e}=87.1^{\circ}, \phi_{e}=-1.0^{\circ}\right), \boldsymbol{M}=\mathbf{0 . 1 7}$.

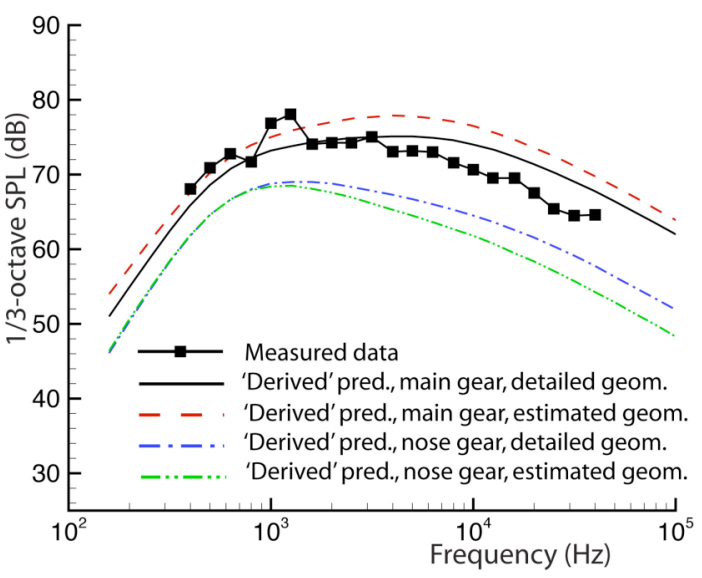

Figure 11. Comparison of "Derived' spectra obtained from Guo method predictions, $\left(\theta_{e}=87.1^{\circ}, \phi_{e}=-1.0^{\circ}\right), \boldsymbol{M}=\mathbf{0 . 1 7}$.

Figure 12 compares "Derived" Guo predictions with data at two different observer locations; 12(a) is for the flyover position and 12(b) is for the forward-sideline position. The total "Derived" spectrum and its three separate frequency component spectra are shown. At both locations, the high frequency component over predicts by about $2-$ $3 \mathrm{~dB}$ at frequencies beyond $10 \mathrm{kHz}$. At frequencies less than $1250 \mathrm{~Hz}$, the predictions compare generally well with the data. At the forward-sideline location (Fig. 12(b)) the low and mid frequency component spectral amplitude is greater than at the flyover location (Fig. 12(a)). As azimuthal angle increases, the contribution of the low- and midfrequency components increase due to the directivity function Eq. (11).

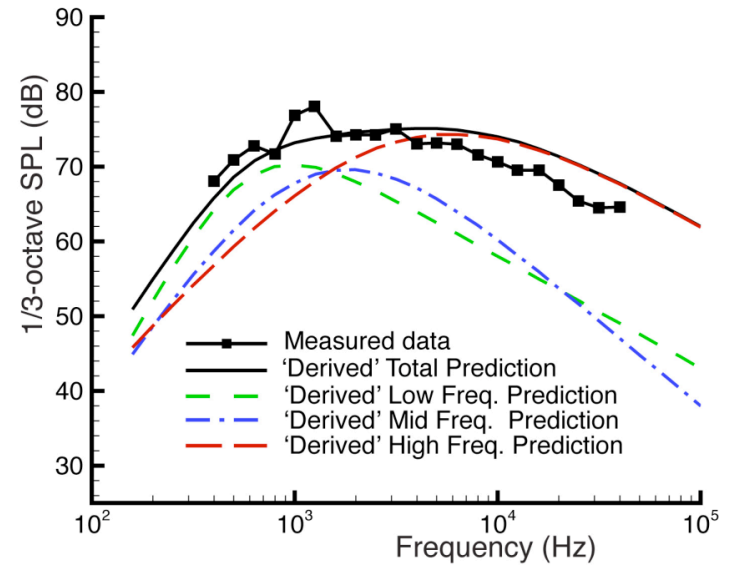

a. Observer position at $\theta_{e}=87.1^{\circ}, \phi_{e}=-1.0^{\circ}$.

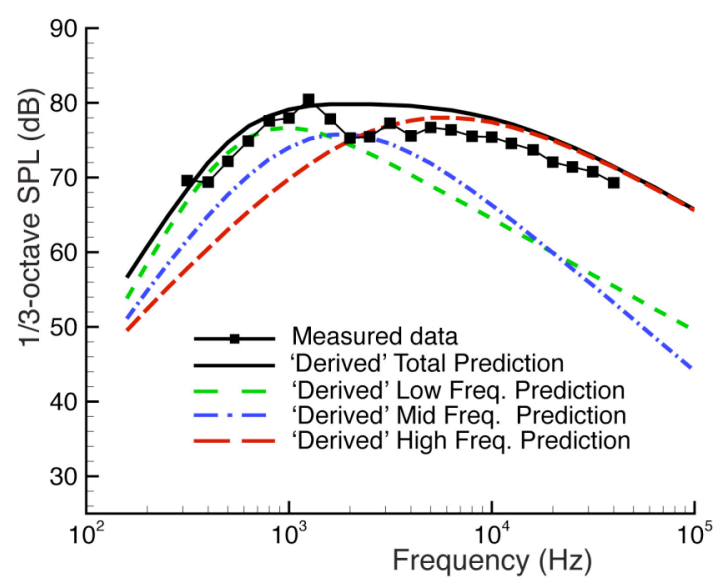

b. Observer position at $\theta_{e}=59.3^{\circ}, \phi_{e}=51.7^{\circ}$.

Figure 12. Comparison of 'Derived' total and component spectra obtained from Guo method predictions with measured data obtained at two different observer locations. $M=0.17$. 
Figure 13 is similar to Fig. 12, but the predictions are from the Fink method. In Fig. 13a, at the flyover location, the predictions compare well over the full frequency range. At this observer location, the wheel component totally dominates the predictions. At the forward-sideline location shown in Fig. 13b, the comparison is considered good at the higher frequencies and only fair for lower frequencies. The wheel component dominates at higher frequencies, whereas the strut component dominates at the lower frequencies. These trends are due to the directivity functions, which are given in Eqs. 4 and 5.

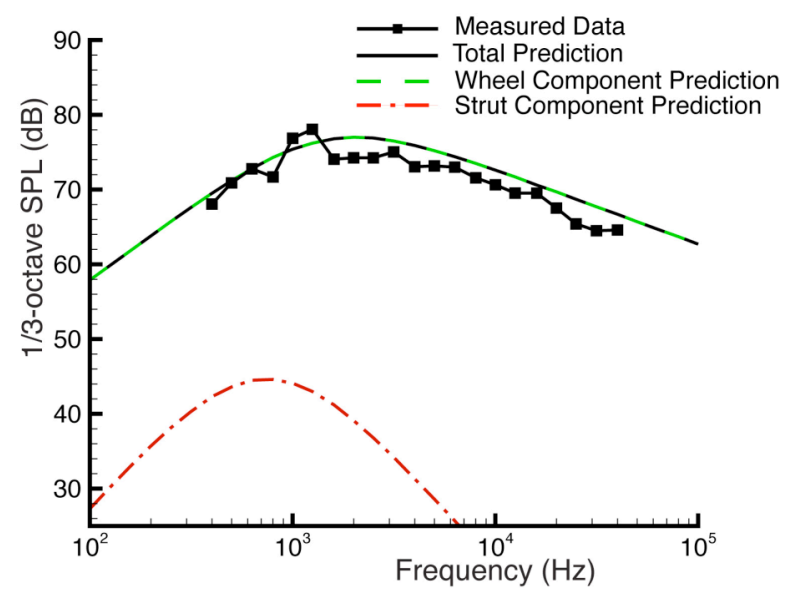

a. Observer position at $\theta_{e}=87.1^{\circ}, \phi_{e}=-1.0^{\circ}$.

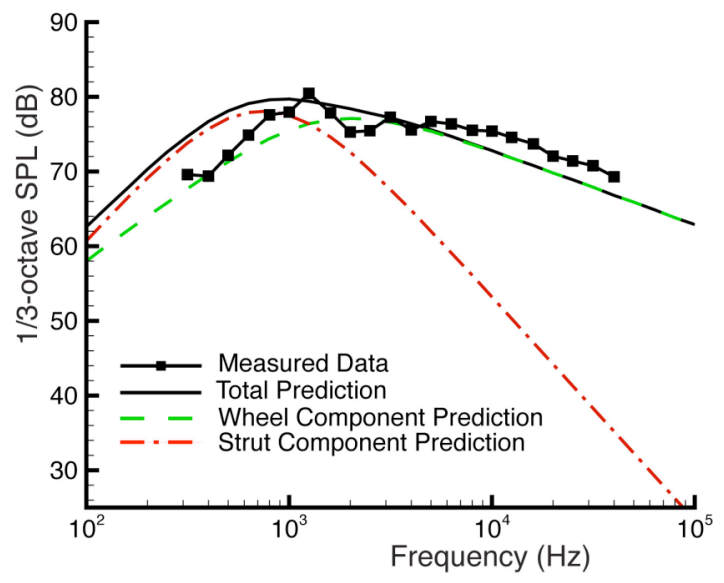
at two different observer locations.

Guo "Derived" and Fink predictions are compared to noise measurements for a range of azimuthal directivity angles in Figs. 14 and 15, respectively. Both methods assume azimuthal symmetry and thus produce the same results at positive and negative azimuthal angles, as indicated in the figures. As noted earlier, both prediction methods are based on data from landing gear that included a door, as does the scale model tested. Measured spectra for both the positive and negative azimuthal angles show the degree of non-symmetry of the noise for the model. At $\phi_{e}=-59.1^{\circ}$ for the observer on the door side, the noise is lower in level compared to that on the non-door side at $\phi_{e}=57.8^{\circ}$. This difference is on the order of $3-5 \mathrm{~dB}$. The Guo "Derived" predictions compare most favorably with the door side data for this azimuth angle, whereas the Fink method over-predicts at all frequencies for the door side. The Fink method shows only fair comparison with the non-door side data. At the larger azimuthal angle, $\phi_{e}=81.6^{\circ}$, the Guo "Derived" predictions compare well in overall spectral shape and amplitude. The Fink prediction compares well only at the highest frequencies for this azimuth angle.

In Figs. 16 and 17, the "Derived" Guo and Fink spectral predictions are compared to measurement for a range of flyover polar angles. The Fink predictions compare remarkably well with measurement in spectral shape and amplitude, particularly at the upstream $\left(\theta_{e}=56.0^{\circ}\right)$ and downstream $\left(\theta_{e}=122.1^{\circ}\right)$ locations. The "Derived" Guo predictions comparisons over-predict by at least $3-5 \mathrm{~dB}$ from the mid to higher frequencies, while comparing well in amplitude at the lower frequencies. The over-prediction could perhaps be attributed to the Guo method being based on full-scale landing gear that represents in the modeling the noise from all the small geometric details (through his complexity factor $\eta$ ), which may be missing from the scale-model gear tested. Note that the Fink predictions vary minimally from upstream to directly underneath $\left(\theta_{e}=56.0^{\circ}\right.$ to $\left.\theta_{e}=87.1^{\circ}\right)$ whereas the Guo method shows an expected shift due to convective amplification. The Fink method includes convective amplification, but the directivity function is predominantly that of the wheel assembly (Eq. (4)), whose directivity effects tend to off-set level increases upstream from convective amplification (Eq. (1)). 


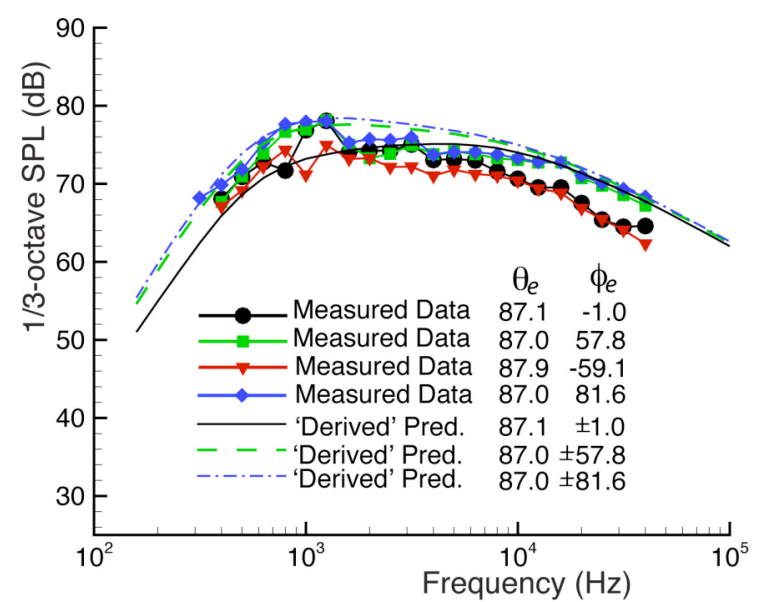

Figure 14. 'Derived' spectra obtained from using the Guo method compared with data for a range of azimuthal directivity angles at a nominal $\theta_{e}=87^{\circ}, \mathbf{M}=\mathbf{0 . 1 7}$.

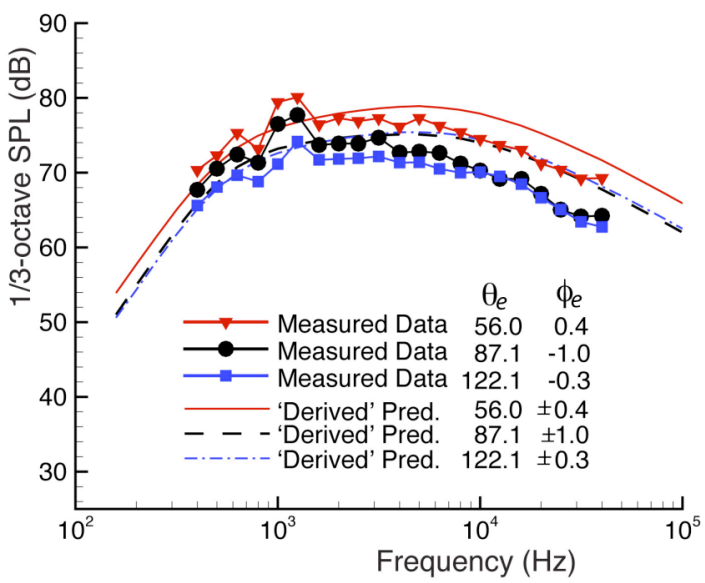

Figure 16. 'Derived' predicted spectra using the Guo method compared with data for range of polar directivity angles, with nominal $\phi_{e}=0^{\circ}, \boldsymbol{M}=\mathbf{0 . 1 7}$.

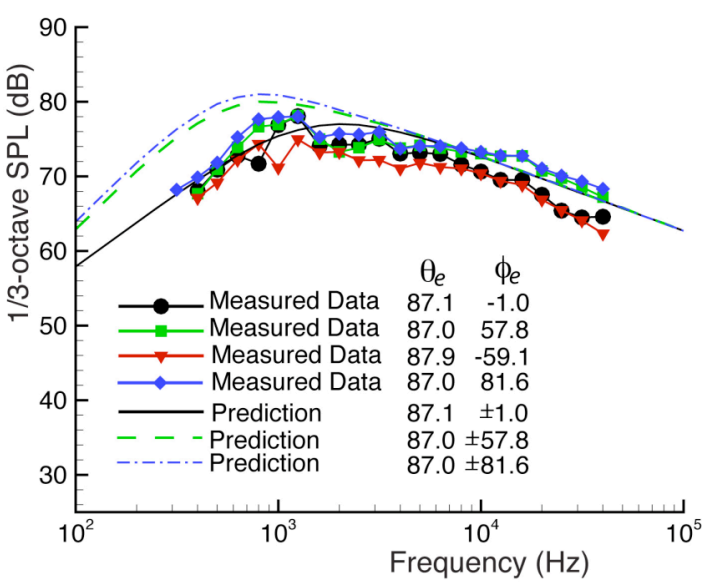

Figure 15. Predicted spectra using the Fink method compared with data for range of azimuthal directivity angles at a nominal $\theta_{e}=87^{\circ}$, $M=\mathbf{0 . 1 7}$.

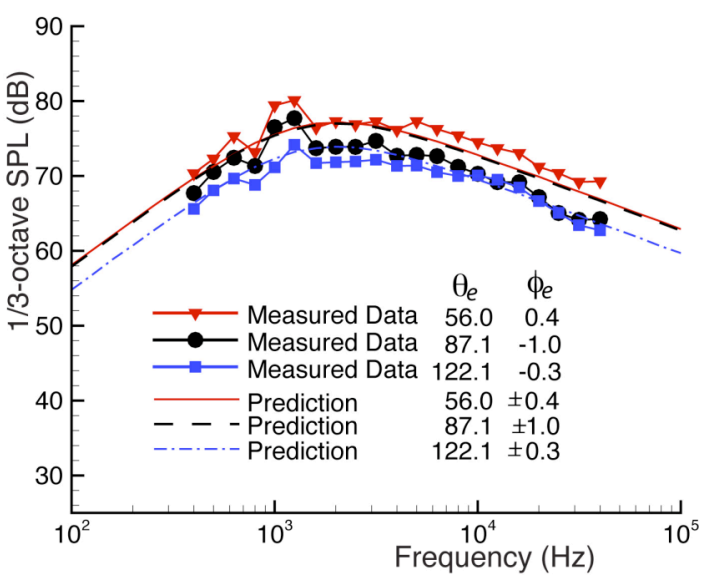

Figure 17. Predicted spectra using the Fink method compared with data for range of polar directivity angles, with nominal $\phi_{e}=0^{\circ}, M=0.17$

\section{Polar/Azimuthal Noise Directivity}

The directivity of landing gear noise is examined using one-third octave SPL contours measured and computed as a function of emission polar and azimuthal angles. A perspective view of the measured noise directivity for the 10 $\mathrm{kHz}$, one-third octave band is shown in Fig. 18. An observer located in the flyover position would be located where the $\mathrm{z}$-axis intersects the bottom center of the hemisphere, near $\theta_{e}=90^{\circ}, \phi_{e}=0^{\circ}$. The small dots on the contour map indicate the microphone measurement locations. The predictions are made at these same locations in order to compare directly with the measurements. To aid in the comparison between the different directivities, the threedimensional hemisphere of Fig. 18 is replaced by two-dimensional projection contour plots, which are shown in Fig. 19 for measured data and in Fig. 20 for each prediction method. The top of each contour is in the direction of flight. A silhouette of the model is shown in Fig. 19 for the $630 \mathrm{~Hz}$ contour and corresponds to an observer directly beneath the gear. The landing gear door is on the left (for which azimuthal angles are negative). 


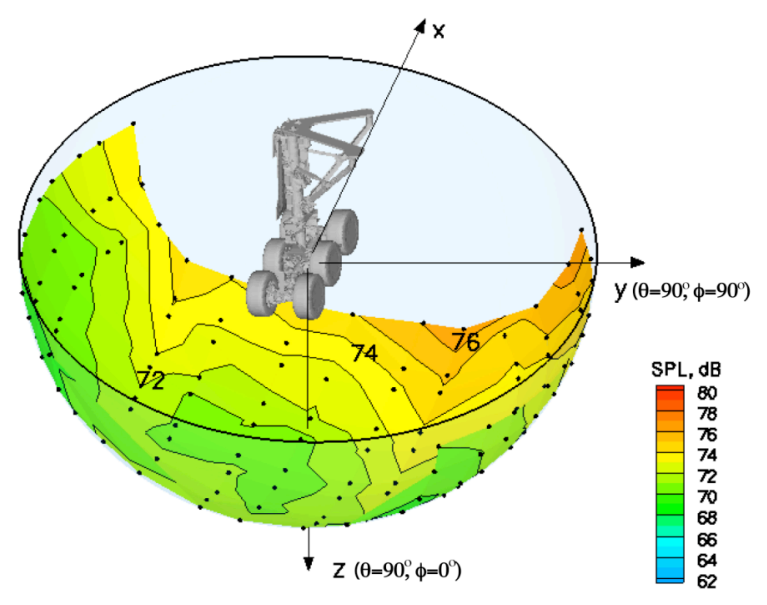

Figure 18. Measured hemispherical noise directivity contours, $M=0.17$. (landing gear not to scale).

Figure 19 shows the measured contours for frequency bands ranging from $630 \mathrm{~Hz}$ through 40 $\mathrm{kHz}$. Figure 20 shows the corresponding predicted contours created using the Fink and Guo methods. Each successive plot represents a doubling in the onethird-octave frequency. The same color scale is used for all the contours in Figs. 19 and 20.

Overall, the predicted contours show a fair comparison with the measured contours. The measured contours have a distinct directionality both in the polar and azimuthal direction. Both prediction methods assume symmetric azimuthal directivity, so differences from one side of the landing gear to the other are not modeled. However, the global azimuthal directivity trends should be. The 'Derived' Guo contours indicate reduced amplitudes in the flyover region, which is also observed in the measured contours. The Fink method does not capture this detail. For the most part, 'Derived' Guo contours capture the overall global directivity character, but tend to over predict the amplitudes, particularly at the higher frequencies. The Fink contours tend to be invariant in azimuthal directivity, but predict the amplitudes better than the Guo method.

For the polar directivity, particularly for $\phi_{e}=0^{\circ}$, the Fink and Guo methods predict similar trends with the largest differences seen in amplitude at the lower frequencies. The measured contours show highest noise levels forward of the landing gear (polar angles less than 90 degrees). The levels decrease by about 5-6
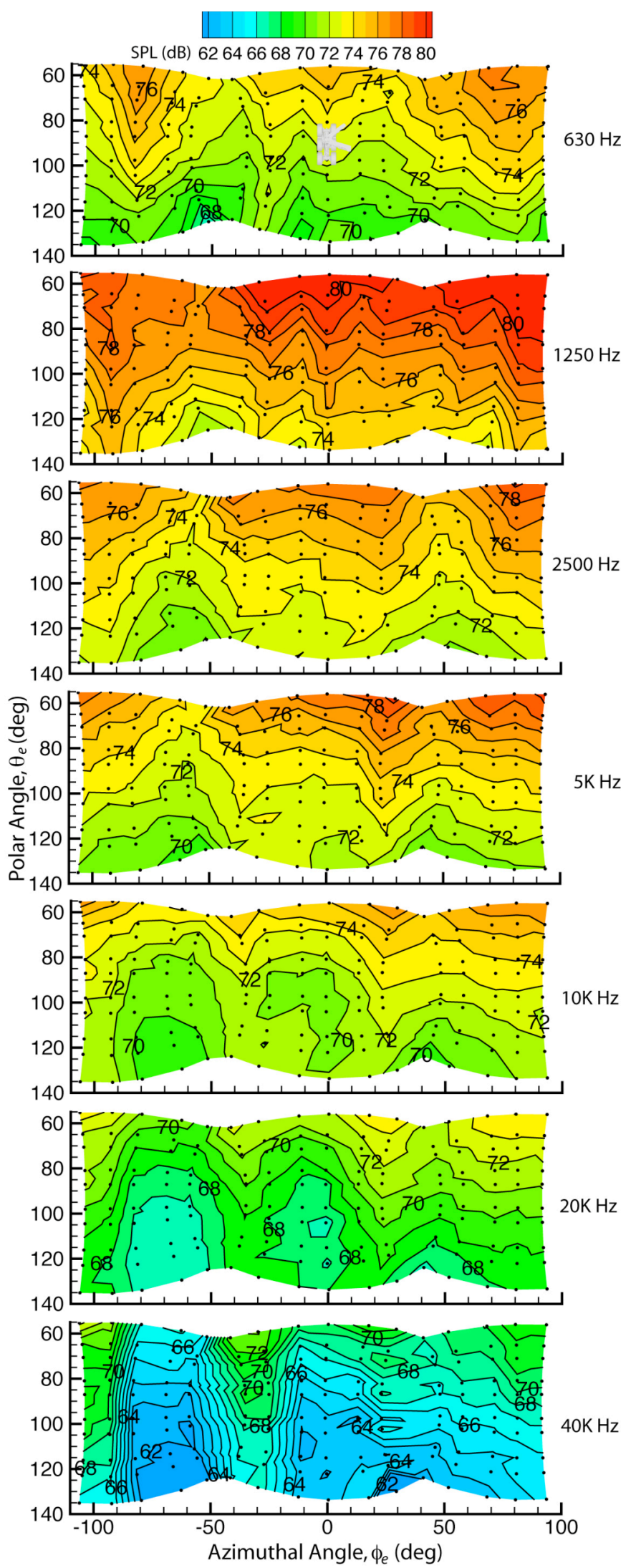

Figure 19. Measured polar/azimuthal noise directivity contours for tested baseline configuration, $M=0.17$. 


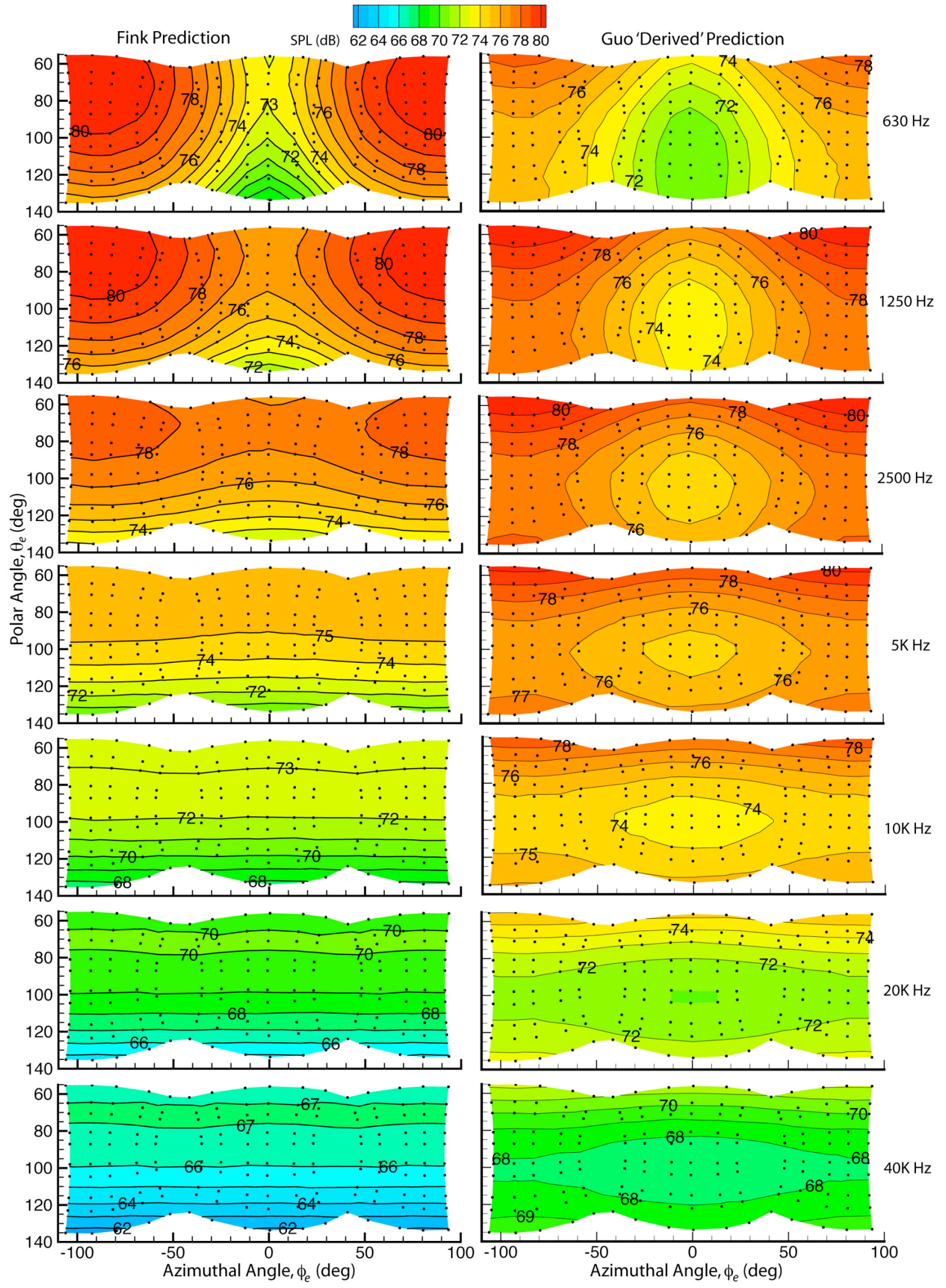

Figure 20. Predicted and 'Derived' polar/azimuthal noise directivity contours, $M=0.17$. 
$\mathrm{dB}$ between the upstream and downstream polar angles for basically all azimuthal angles. This is consistent with the full-scale landing gear measurements of Dobrzynski et $\mathrm{al}^{6}$. Both the Fink and Guo methods predict this trend along the flight path $\left(\phi_{e}=0^{\circ}\right)$, with the Fink results matching the absolute amplitudes more consistently than that of Guo.

Based on A-weighting noise metric (dBA), the most important frequencies for community noise concerns are typically those greater than $1000 \mathrm{~Hz}$ full-scale, which is about $15 \mathrm{kHz}$ model scale. Hence data and predictions at 20 $\mathrm{kHz}$ and $40 \mathrm{kHz}$ are most relevant for community noise metrics. At these frequencies, the Fink predictions compare slightly better in level with the measurements than the 'Derived' Guo predictions; whereas, that of Guo method compares slightly better in directivity, since it indicates a reduced noise level region directly under the gear.

\section{Conclusions}

NASA's ANOPP offers two empirically based methods to predict landing gear noise: the "Fink" method and the "Guo" method. The "Guo" method was developed almost exclusively using full-scale landing gear data. The "Fink" method was developed using both model and full-scale data. Both have previously been shown to compare well with full-scale noise data. Application of these methods to a model-scale landing gear was investigated by comparing predicted results from each method with results from a high-fidelity, 6.3\%-scale, Boeing 777 main landing gear. The Fink method was found to follow Strouhal scaling, and, hence, employed the scale model geometry as input. The "Guo" method, although largely based on Strouhal scaling approaches, uses narrowband spectrum functions and a "complexity factor" that prevents its use for arbitrary landing gear sizes. A method was developed to create "Derived" model-scale prediction from full-scale predictions that could be correctly compared with model data.

The measured polar and azimuthal directivity spectra and contour plots reveal distinctive directivity patterns, with higher levels of noise directed forward and away from the door side of the landing gear. The Fink and Guo "Derived" predictions show good comparisons for flyover polar angles and fair comparisons for overall directivity, particularly at the larger azimuthal angles. These comparisons suggest that noise measured for a high-fidelity, scalemodel landing gear is representative of that found for full-scale landing gear, since the prediction methods were developed and compared well with full-scale data. The noise directivity models in both ANOPP prediction methods can now be improved due to the availability of extensive measurements.

\section{Acknowledgments}

The authors wish to thank Dr. Yueping Guo of Boeing Phantom Works for providing the full-scale Boeing 777 landing gear geometry input to the Guo method.

\section{References}

${ }^{1}$ Zorumski, W.E., “Aircraft Noise Prediction Program Theoretical Manual, Parts 1 and 2,” NASA TM-83199-PT-1 and PT-2, National Aeronautics and Space Administration, Langley Research Center, Hampton, VA., February 1982.

${ }^{2}$ Hubbard, H., "Aeroacoustic Research Facilities at NASA Langley Research Center," NASA Technical Memorandum 84585 , 1983.

${ }^{3}$ Heller, H., and Dobrzynski, W., "Sound Radiation from Aircraft Wheel-Well / Landing-Gear Configurations," Journal of Aircraft, vol. 14, no. 8, pp. 768 - 774, 1977.

${ }^{4}$ Fink, M., "Noise Component Method for Airframe Noise," Journal of Aircraft, vol. 16, no. 10, pp. 659-665, 1979.

${ }^{5}$ Fink, M. R., “Airframe Noise Prediction Method," FAA-RD-77-29, U.S. Department of Transportation, Federal Aviation Administration, March, 1977.

${ }^{6}$ Dobrzynski, W., and Buchholz, H., "Full-Scale Noise Testing on Airbus Landing Gears in the German Dutch Wind Tunnel," AIAA Paper 97-1597, $3^{\text {rd }}$ AIAA/CEAS Aeroacoustics Conference, Atlanta, GA, 1997.

${ }^{7}$ Dobrzynski, W., Chow, L.C., Guion, P., and Shiells, D., "A European Study on Landing Gear Airframe Noise Sources," AIAA Paper 2000-1971, $6^{\text {th }}$ AIAA/CEAS Aeroacoustics Conference, Lahaina, HI, 2000. 
${ }^{8}$ Dobrzynski, W., Schoning, B., Chow, L.C., Wood, C., Smith, M., and Seror, C., "Design and Testing of Low Noise Landing Gears," AIAA Paper 2005-3008, $11^{\text {th }}$ AIAA/CEAS Aeroacoustics Conference, Monterey, California, May 2005.

${ }^{9}$ Michel, U., and Qiao, W., "Directivity of Landing-Gear Noise Based on Flyover Measurements," AIAA Paper 99-1956, $5^{\text {th }}$ AIAA/CEAS Aeroacoustics Conference, Bellevue, WA, 1999.

${ }^{10}$ Jaeger, S., Burnside, N., Soderman, P., Horne, W.C., and James, K., "Aeroacoustic Study of an Isolated, High-Fidelity, 26\%-Scale Landing Gear Model," NASA Technical Paper 2004-212815, November, 2004.

${ }^{11}$ Horne, W.C., Burnside, N., Soderman, P., Jaeger, S., Reinero, B., James, K., and Arledge, T., "Aeroacoustic Study of a 26\%-Scale Semispan Model of a Boeing 777 Wing in the NASA Ames 40- by 80-Foot Wind Tunnel," NASA Technical Paper 2004-212802, October, 2004.

${ }^{12}$ Stoker, R., and Sen, R., “An Experimental Investigation of Airframe Noise Using a Model-Scale Boeing 777,” AIAA Paper 2001-0987, 39 $9^{\text {th }}$ AIAA Aerospace Sciences Meeting, Reno, NV, 2001.

${ }^{13}$ Humphreys, W., and Brooks, T., "Noise Spectra and Directivity For a Scale-Model Landing Gear," AIAA Paper 20073458, $13^{\text {th }}$ AIAA/CEAS Aeroacoustics Conference, Rome, Italy, May 21-23, 2007.

${ }^{14}$ Smith, M., and Chow, L., "Validation of a Prediction Model for Aerodynamic Noise from Aircraft Landing Gear", AIAA Paper 2002-2581, $8^{\text {th }}$ AIAA/CEAS Aeroacoustics Conference, Breckenridge, CO, 2002.

${ }^{15}$ Molin, N., Piet, J., Chow, L., Smith, M., Dobrzynski, W., Seror, C., "Prediction of Low Noise Aircraft Landing Gears and Comparison With Test Results," AIAA Paper 2006-2623, $12^{\text {th }}$ AIAA/CEAS Aeroacoustics Conference, Cambridge, Massachusetts, May 2006.

${ }^{16}$ Guo, Y., "Empirical Prediction of Aircraft Landing Gear Noise," NASA Contractor Report 2005-213780, July, 2005.

${ }^{17}$ Guo, Y., "A Semi-Empirical Model for Aircraft Landing Gear Noise Prediction," AIAA Paper 2006-2627, 12 $2^{\text {th }}$ AIAA/CEAS Aeroacoustics Conference, Cambridge, Massachusetts, May, 2006.

${ }^{18}$ Guo, Y., “An Improved Landing Gear Noise Prediction Scheme,” NASA Informal Contractor Report, NAS1-NN104AA11B Task NNL06AB63T.

${ }^{19}$ Hedges, L., Travin, A., and Spalart, P., "Detached-Eddy Simulations Over a Simplified Landing Gear," Journal of Fluids Engineering, vol. 124, no. 2, pp. 413-423, 2002.

${ }^{20}$ Seror, C., Saguaut, P., and Belanger, A., “A Numerical Aeroacoustics Analysis of a Detailed Landing Gear,” AIAA 20042884, $10^{\text {th }}$ AIAA/CEAS Aeroacoustics Conference, Manchester, U.K., 2004.

${ }^{21}$ Lockard, D. and Khorrami, M., “Aeroacoustic Analysis of a Simplified Landing Gear," AIAA Paper 2004-2887, 10 AIAA/CEAS Aeroacoustics Conference, Manchester, U.K., 2004.

${ }^{22}$ Sen, R. Hardy, B., Yamamoto, K., Guo, Y, and Miller, G., “Airframe Noise Sub-component Definition and Model,”,Boeing Commercial Airplane Company, NASA Contractor Informal Report, January 2000.

${ }^{23}$ Guo, Y., "Gearnoise06 User Manual,” NASA Informal Contractor Document, NAS1-NN104AA11B Task NNL06AB63T.

${ }^{24}$ Brooks, T. F., Pope, D., and Marcolini, M. A., “Airfoil Self-Noise and Prediction”, NASA RP 1218, July 1989.

${ }^{25}$ Schmitz, F. H., Boxwell, D.A., Splettstoesser, W.R., and Schultz, K.J., "Model-Rotor High-Speed Impulsive Noise: Full Scale Comparisons and Parametric Variations," Vertica, vol. 8, no. 4, 1984, pp. 395-422.

${ }^{26}$ Brooks, T. F., Jolly, J.R.,Jr., and Marcolini, M. A., "Helicopter Main-rotor Noise - Determination of Source Contributions Using Scaled Model Data," NASA TP 2825, August 1988. 